\title{
Klinische Beobachtung des Einflusses der Avertinrektainarkose auf die Alkalireserve des Bluts und den Blutzucker.
}

\author{
Von \\ Toyoya Nakamura. \\ (中 村 䅱 彌) \\ (Aus der Chirurgischen Klinik der Kaiserlichen TohokuUniver- \\ sität zu Sendai. Torstand: Prof. Dr. Sh. Sekiguchi.)
}

\section{Einleitung.}

Infolge häufiger Anwendung der Avertinrektalnarkose wird sie neuerdings von verschiedenen klinischen Standpunkten aus erforscht. Ihre Schwäche, worin sie den Inhalationsnarkosen nachsteht, liegt ausschliesslich darin, dass sie keine willkürliche Unterbrechung zulässt. Deshalb ist es sehr wichtig festzustellen, inwieweit die Protrahierung der Avertinrektalnarkose von der Menge des Mittels abhängt. Weiter ist es klinisch von Bedeutung, unter den verschiedenen, dabei im Blut auftretenden Erscheinungen vor allem den Einfluss auf das Säurebasengleich gewicht und den Blutzucker zu erkennen. Hierüber wird nur sehr selten berichtet. Deshalb habe ich in vorliegender Arbeit den Einfluss der Avertinrektalnarkose auf die Alkalireserve des Bluts und den Blutzucker untersucht und zugleich auch den klinischen Verlauf beobachtet.

\section{Erster Teil. Alkalireserve des Bluts.}

I. Methodik.

(1) Versuchsmethode. Aus der kubitalen Vene entnahm ich Blut, ohne die. Blutstauung herbeiführen zu lassen. Nach Van Slykes Methode bestimmte ich dann die Alkalireserve im Vollblut. 2 Stunden vor der Narkose und 30 Minuten, 3, 6, 24, 48, Stunden, 4 und 7 Tage nach der Narkose, also 8 Male entnahm ich Blut und bestimmte die Alkalireserve des Bluts. 30 Minuten nach 
Avertinzufuhr war das Mittel schon fast bis zu $90 \%$ resorbiert, und Atmung, Puls und Blutdruck waren auch schon wieder fast beständig geworden. In meinem Versuch ist dies gerade der Zeitpunkt von der Operation. 3 Stunden nach der Narkose blieben noch viele Fälle in tiefer Narkose, wo die Operation schon vollendet war. Nach 6 Stunden ist das Stadium des Nachschlafs oder des Erwachens, und nach 24 Stunden waren die Fälle teils schon wieder normal, teils schienen sie sich noch ganz müde zu sein.

(2) Narkosenmethode. Je nach dem Allgemeinzustand und dem Grad der Operation benutzte ich $0,08-0,17 \mathrm{~g} 2,5 \%$ iger Avertinwasserlösung pro kg. In den meisten Fällen trat Narkose schon 5-15 Minuten nach dem Einlauf ein. Am Vorabend der Narkose spülte ich den Darm mit Seifenlösung und am nächsten Morgen mit Glyzerin.

(3) Versuchsfälle. Zum Versuch benutzte ich 66 Fälle, die sich alle einer chirurgischen Operation unterzogen, und zur Kontrolle 9 Fälle, die unter Lokalnarkose operiert wurden.

\section{Ergebnisse.}

\section{(1) Alkalireserve des Bluts bei chirurgischen Kranken.}

Man glaubt allgemein, dass bei einer gewissen Reihe chirurgischer Krankheiten die Alkalireserve des Bluts mehr oder weniger zur Abnahme neigt. Unter meinen Versuchsfällen aber zeigte die Alkalireserve, obwohl alle 75 Fälle $\mathrm{zu}$ den verschiedenen chirurgischen Krankheiten gehörten, wie aus Tabelle 1 und 2 zu ersehen, beim 4 . Fall ihren Maximalwert, d.h. 54,2 Vol.\%, und beim 10. ihren Minimalwert, d.h. 42,1 Vol.\%; ihr Durchschnittswert betrug 48,3 Vol.\%. Bei der Eiplepsie und im Intervall-Appendizitis und beiHernia war sie beinahe ebenso gut wie bei Gesunden; bei diesen oben genannten drei Krankheiten zeigte sie keinen grossen Unterschied, d. h. sie betrug beim einen wie dem anderen mehr als $48 \mathrm{Vol} \%$. Fast ebenso stand es mit den anderen Krankheiten, aber bei tuberkulösen oder anderen schweren Krankheiten sank sie etwas unter diesen Durchschnittswert. Aber klinisch ist die Alkalireserve bei Gesunden nicht immer hoch und bei Kranken auch nicht immer tief. Bei 56. und 10. Fall blieb sie auf 42 Vol.\% und etwas darüber, während sie beim 51 . und 20 . mehr als 48 Vol.\% betrug. Obgleich die Alkalireserve des Bluts jedenfalls auch vor der Operation, wie bekannt, durch verschiedene Einflüsse abnimmt, so lässt sich doch nicht leicht bestimmen, woher das kommt. Also zeigte die Alkalireserve im allgemeinen bei vielen nicht so bedeutenden Krankheiten keine nennenswerte Abweichung, wälrend die schweren Erkrankung wie Tuberkulose merkliche Schwankungen aufweisen können. 
Tabelle

$\mathrm{CO}_{2}$-Gehalt im

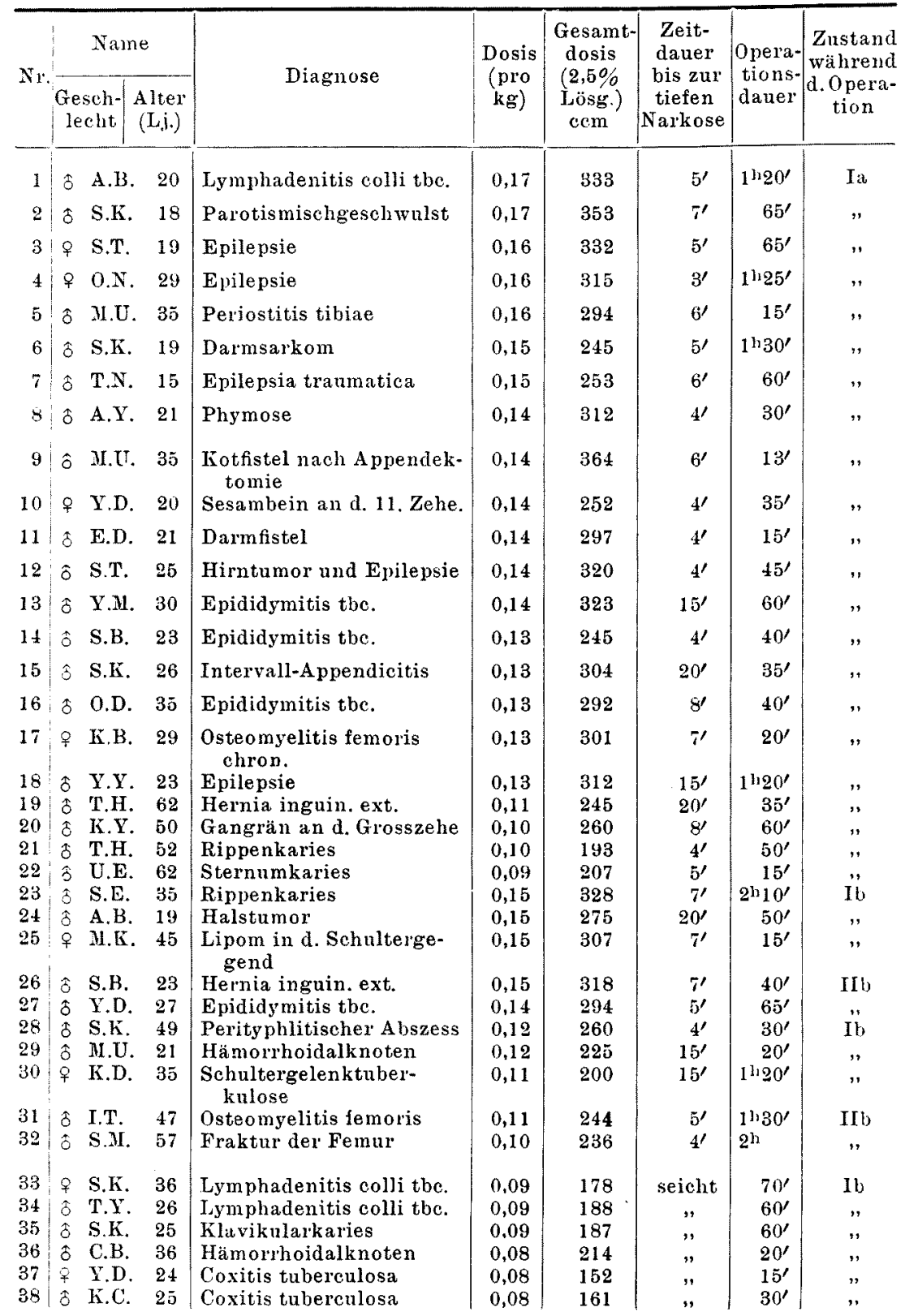


1.

Blut.

\begin{tabular}{|c|c|c|c|c|c|c|c|c|c|c|c|}
\hline \multirow{2}{*}{$\begin{array}{c}\text { Inhala- } \\
\text { tions- } \\
\text { narkose } \\
\text { (̈̈ther) }\end{array}$} & \multirow{2}{*}{$\begin{array}{l}\text { Atem- } \\
\text { störung }\end{array}$} & \multirow{2}{*}{$\begin{array}{c}\text { Zeitdauer } \\
\text { bis zam } \\
\text { Erwachen }\end{array}$} & \multirow{2}{*}{ Operation } & \multirow{2}{*}{$\begin{array}{c}\text { Vor d. } \\
\text { Nar- } \\
\text { kose } \\
\text { (Vol. } \\
\% \text { ) }\end{array}$} & \multicolumn{7}{|c|}{ Nach d. Narkose } \\
\hline & & & & & $\mid \begin{array}{c}30 \\
\text { M in. }\end{array}$ & $\begin{array}{c}3 \\
\text { Std }\end{array}$ & $\begin{array}{c}6 \\
\text { Std. }\end{array}$ & $\begin{array}{c}24 \\
\text { Std. }\end{array}$ & $\begin{array}{c}48 \\
\text { Std. }\end{array}$ & $\begin{array}{c}4 \\
\text { Tag. }\end{array}$ & $\begin{array}{c}7 \\
\text { Tag. }\end{array}$ \\
\hline- & - & $11^{\mathrm{b}}$ & Exstirpation & 49,6 & 46,8 & & 40,1 & 49,6 & 49,8 & 50,3 & 50,6 \\
\hline- & + & $20^{h}$ & " & 48,6 & 48,5 & & 39,1 & 44,8 & 50,4 & 47,3 & 47,0 \\
\hline- & - & $11^{\mathrm{n}} 30^{\prime}$ & Trepanation & 47,5 & 48,7 & & 34,6 & 45,4 & 53,9 & 46,9 & 47,6 \\
\hline- & - & $18^{\mathrm{h}}$ & $"$ & 54,2 & 52,6 & & 46,0 & 45,9 & 50,6 & 49,5 & $52, \tilde{0}$ \\
\hline- & - & $11^{\mathrm{h}}$ & Inzision & 49,2 & 49,9 & & 42,3 & 43,8 & 48,9 & 48,4 & 43,4 \\
\hline - & - & $11^{\mathrm{b}} 30^{\prime}$ & Exstirpation & 50,5 & 49,5 & & 41,0 & 42,9 & 45,6 & 53,9 & 49,1 \\
\hline- & - & $6^{\text {h }}$ & Trepanation & 50,3 & 46,4 & & 35,5 & 41,6 & 50,8 & 50,3 & 49,0 \\
\hline - & + & $11^{\mathrm{h}} 30^{\prime}$ & $\begin{array}{c}\text { Plastische } \\
\text { Operation }\end{array}$ & 45,5 & 40,8 & & 41,4 & 41,4 & & 40,4 & 45,7 \\
\hline- & - & $14^{\mathrm{h}}$ & Appendek tomie & 49,7 & 51,5 & & 43,9 & 40,0 & 47,2 & 47,4 & 48,8 \\
\hline- & + & $14^{\mathrm{ln}}$ & Exstirpation & 42,1 & 43,1 & & 40,0 & 40,9 & 45,3 & 45,7 & 43,0 \\
\hline- & + & $6^{\text {h }} 30^{\prime}$ & Inzision & 51,6 & 46,3 & & 41,3 & 49,3 & 51,2 & 51,2 & 50,7 \\
\hline- & - & $14^{\mathrm{h}} 30^{\prime}$ & Trepanation & 49,9 & 49,1 & 45,9 & 39,7 & 47,7 & 50,7 & 47,1 & 47,6 \\
\hline- & - & $13^{h}$ & Kastration & 47,0 & 48,1 & & 41,2 & 49,8 & 50,7 & 43,1 & 43,4 \\
\hline- & - & $6^{h}$ & $"$ & $4 \overline{0}, 7$ & 47,5 & & 38,4 & 37,8 & 48,6 & 48,3 & 46,7 \\
\hline- & - & $8^{\text {h }}$ & Appendektomie & 50,0 & 50,6 & & 44,9 & 45,3 & 47,3 & 42,1 & 45,8 \\
\hline- & - & $8^{h}$ & Kastration & 50,2 & 46,2 & & 36,4 & 38,7 & 47,8 & 44,0 & 48,6 \\
\hline- & - & $11^{\mathrm{h}}$ & Aufmeisselung & 49,8 & 47,9 & 40,4 & 38,2 & 43,8 & 40,0 & 45,4 & 45,7 \\
\hline- & - & $8 \mathrm{l}$ & Trep & 46,4 & 45,3 & & 42,8 & 46,5 & 49,3 & 49,1 & 46,7 \\
\hline - & + & $14^{\mathrm{h}}$ & Her & 51,7 & $\mid 49,8$ & 47,8 & 45,2 & 41,3 & 49,5 & 49,6 & 50,0 \\
\hline- & - & $7^{\mathrm{h}}$ & $A m p$ & 49,2 & 49,2 & 48,3 & 44,0 & 46,7 & 51,2 & 50,3 & 50 \\
\hline- & + & $12 \mathrm{~h}$ & ktion & 51,6 & $\mid 52,4$ & & 46,7 & $52,5 \mid$ & 54,4 & 52,8 & 51 \\
\hline - & + & 15 & Inz & 48,2 & 46,4 & & 37,7 & $48,0 \mid$ & 42,8 & 50,2 & 50 \\
\hline- & - & 12 & $\operatorname{Rin}$ & 49,2 & $|48,7|$ & & 43,0 & $|49,7|$ & 49,3 & 49,8 & 50 \\
\hline - & - & $16^{\mathrm{l}}$ & Exstirpation & 49,2 & 46,4 & 43,4 & 39,5 & 49,1 & $\mid 49,2$ & 49,7 & 49 \\
\hline 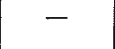 & 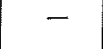 & $12^{\mathrm{h}}$ & $"$ & 50,2 & 47,3 & 47,3 & 43,3 & 50,6 & 50,2 & 51,9 & 51,9 \\
\hline- & - & $6^{\text {h }}$ & Herniotomie & 49,3 & 50,6 & 48,9 & 43,0 & 49,6 & 49,0 & 51,0 & 47,8 \\
\hline - & 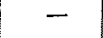 & $10^{\mathrm{h}}$ & & 47,2 & 47,1 & & 38,5 & $47,5 \mid$ & $48,9 \mid$ & 47,9 & 44,2 \\
\hline - & - & $7 \mathrm{~h}$ & tomie & 49,7 & $46,1 \mid$ & & 39,6 & 41,0 & 46,8 & 43,3 & 44 \\
\hline - & - & & Kat & 45,0 & 44,7 & & $37,0 \mid$ & 47,4 & 50,0 & 50,0 & 49,4 \\
\hline & & 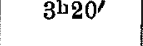 & Exarticulation & 46,4 & 42,3 & & 31,1 & 41,3 & 48,0 & 46,6 & 48,1 \\
\hline- & + & $10^{\mathrm{h}}$ & lung & 51,6 & 45,7 & & 43,8 & 45,7 & 50,0 & 49,3 & 47,4 \\
\hline & + & $12^{\mathrm{h}}$ & $\begin{array}{l}\text { Plastische } \\
\text { Operation }\end{array}$ & 51,3 & 50,0 & & 38,2 & 41,0 & 46,5 & 46,5 & 50,5 \\
\hline - & - & $4^{b} 30^{\prime}$ & Exstirpation & 49,0 & $|48,7|$ & & 43,2 & 50,7 & $|49,7|$ & 52,7 & 51,2 \\
\hline 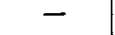 & & & & 46,3 & $|45,4|$ & & 44,4 & 44,5 & 47,2 & 47,1 & \\
\hline- & 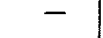 & $8 \mathrm{~h}$ & & 43,5 & 42,1 & & 35,4 & 45,8 & 45,8 & 44,2 & 46,3 \\
\hline- & - & 3b & Kauterisation & 45,4 & 44,2 & & 43,5 & 43,4 & 48,1 & 47,1 & 49,4 \\
\hline - & - & $5^{\mathrm{h} 3} 30^{\prime}$ & Punktion & 48,0 & $|43,0|$ & & 43,1 & 49,6 & 50,6 & 44,5 & 47,2 \\
\hline & - & $5^{\mathbf{b}} 30^{\prime}$ & $"$ & 50,0 & $|45,0|$ & & $|37,4|$ & 49,6 & 54,7 & 49,2 & 51,0 \\
\hline
\end{tabular}




\begin{tabular}{|c|c|c|c|c|c|c|c|c|}
\hline \multirow{2}{*}{$\mathrm{Nr}$} & \multicolumn{2}{|c|}{ Name } & \multirow{2}{*}{ Diaggose } & \multirow{2}{*}{$\begin{array}{c}\text { Dosis } \\
\text { (pro } \\
\mathrm{kg} \text { ) }\end{array}$} & \multirow{2}{*}{$\begin{array}{l}\text { Gesamt- } \\
\text { dosis } \\
(2,5 \% \\
\text { l,ösg. }) \\
\text { ccm }\end{array}$} & \multirow{2}{*}{$\begin{array}{c}\text { Zeit- } \\
\text { dauer } \\
\text { bis zur } \\
\text { tiefen } \\
\text { Narkose }\end{array}$} & \multirow{2}{*}{$\begin{array}{l}\text { Opera- } \\
\text { tions- } \\
\text { dauer }\end{array}$} & \multirow{2}{*}{$\begin{array}{l}\text { Zustand } \\
\text { wählend } \\
\text { d. Opera- } \\
\text { tion }\end{array}$} \\
\hline & $\mid$ & $\begin{array}{l}\text { Alter } \\
(\mathrm{L} . \mathrm{i} .)\end{array}$ & & & & & & \\
\hline 39 & \& $\mathbf{M} . \mathrm{K}$ & 42 & $\begin{array}{l}\text { Lipom in d. Schulter- } \\
\text { gegend }\end{array}$ & 0,08 & 191 & seicht & $3^{\mathrm{h}} 30^{\prime}$ & $\mathrm{Ib}$ \\
\hline 40 & 8 H.K. & 18 & $\begin{array}{l}\text { Osgood-Schlat ter'sche } \\
\text { Krankheit }\end{array}$ & 0,15 & 275 & $"$ & $33^{\prime}$ & III \\
\hline 41 & o T.G. & . 24 & Bauchwandhernie & 0,15 & 244 & $5 \prime$ & $50^{\prime}$ & $"$ \\
\hline 42 & o M.B. & 23 & Hernia inguin. ext. & 0,15 & 356 & $5^{\prime}$ & $35^{\prime}$ & $n$ \\
\hline 43 & $\delta$ L.K. & · 22 & Darmfistel & 0,15 & 344 & $4^{\prime}$ & $1130^{\prime}$ & ", \\
\hline 44 & o T.H. & 33 & Rippenkaries & 0,14 & 334 & $6^{\prime}$ & $60^{\prime}$ & " \\
\hline $4 \tilde{5}$ & o $\mathrm{Y} . \mathrm{M}$. & 30 & Epididymitis tbc. & 0,12 & 273 & $16^{\prime}$ & $60^{\prime}$ & , \\
\hline 46 & 8. K.N & 31 & Intervall-A ppendicitis & 0,12 & 266 & $12^{\prime}$ & $50^{\prime}$ & $"$ \\
\hline 47 & of K.Y. & . 30 & Bronchialfistel & 0,12 & 264 & $20^{\prime}$ & $40^{\prime}$ & $"$ \\
\hline 48 & o M.U. & 15 & Wolfsrachen & 0,12 & 220 & $8^{\prime}$ & $70^{\prime}$ & $"$ \\
\hline 49 & o A.B. & 18 & $\begin{array}{l}\text { Fistelbildung nach Ap- } \\
\text { pendektomie }\end{array}$ & 0,12 & 219 & $12^{\prime}$ & $1^{11} 20^{\prime}$ & , \\
\hline 50 & \& S.D. & 21 & Epilepsie & 0,11 & 258 & $30^{\prime}$ & $2^{\mathrm{h}} 5^{\prime}$ & $"$ \\
\hline 51 & o H.N. & .25 & Lymphadenitis colli tbe. & 0,10 & 238 & $7^{\prime}$ & $30^{\prime}$ & $"$ \\
\hline 52 & o M.R. & - 23 & Penisfistel & 0,10 & 229 & seicht & $30^{\prime}$ & $"$ \\
\hline 53 & $\delta$ C.B. & 36 & Hämorrhoidalknoten & 0,09 & 174 & $30^{\prime}$ & $2 \mathrm{~h} 30^{\prime}$ & $"$ \\
\hline 54 & o M.U. & 25 & Lymphadenitis colli tbc. & 0,15 & 336 & $5^{\prime}$ & $45^{\prime}$ & II a \\
\hline 55 & o S.D. & 23 & Hernia inguin. ext. & 0,15 & 322 & $5^{\prime}$ & $30^{\prime}$ & " \\
\hline 56 . & 우 S.Z. & 20 & Epilepsie & 0,14 & 318 & $8^{\prime}$ & $70^{\prime}$ & $"$ \\
\hline 57 & o M.K. & 42 & Rippenkaries & 0,14 & 327 & $4^{\prime}$ & $1^{\mathrm{h}} 30^{\prime} \mid$ & " \\
\hline 58 & \& A.B. & . 19 & Halstumor & 0,13 & 273 & $20^{\prime}$ & $60^{\prime}$ & $"$ \\
\hline 59 & \& K.T. & . 20 & Lymphadenitis colli tbe. & 0,12 & 236 & $30^{\prime}$ & $2 \mathrm{~b} 40^{\prime}$ & $"$ \\
\hline 60 & S.K. & 25 & Senkungsabszess & 0,11 & 233 & $5^{\prime}$ & $30^{\prime}$ & " \\
\hline 61 & ô Y.H. & . 57 & Parotistumor & 0,10 & 282 & $10^{\prime}$ & $60^{\prime}$ & $"$ \\
\hline 62 & o K.Y. & . 68 & Lymphadenitis colli tbc. & 0,10 & 229 & $6^{\prime}$ & $2^{\ln } 10^{\prime}$ & $"$ \\
\hline 63 & o S.E. & 22 & $\begin{array}{l}\text { Fistelbildung nach Appen- } \\
\text { dek tomie }\end{array}$ & 0,10 & 191 & $13^{\prime}$ & $20^{\prime}$ & $"$ \\
\hline 64 & o S.T. & 37 & Peritonitis tbc. & 0,10 & 165 & $15^{\prime}$ & $35^{\prime}$ & " \\
\hline 65 & $\delta$ Y,S. & 32 & $\begin{array}{l}\text { Epididymitis tbc. mit } \\
\text { Fistelbildung }\end{array}$ & 0,09 & 173 & seicht & $45^{\prime}$ & $"$ \\
\hline 66 & $\hat{o}$ K.D. & - 16 & Rippenkaries & 0,08 & 107 & seicht & $\left|1^{1} 30^{\prime}\right|$ & $"$ \\
\hline
\end{tabular}

Anmerkungen.

Ta......Die Gruppe, die während der Operation ganz ruhig war.

Ib...... Die Gruppe, die zwar am Anfang der Operation ganz ruhig war, am Ende aber etwas stöhnte.

IIa..... Die Gruppe, die schon vom Anfang der Operation an unruhig war, aber keine Inhalationsnarkose bedarf. 


\begin{tabular}{|c|c|c|c|c|c|c|c|c|c|c|c|}
\hline \multirow{2}{*}{$\begin{array}{l}\text { Inhala- } \\
\text { tions- } \\
\text { narkose } \\
\text { (Ather) }\end{array}$} & \multirow{2}{*}{$\begin{array}{l}\text { Atem- } \\
\text { störung }\end{array}$} & \multirow{2}{*}{$\begin{array}{c}\text { Zejtdauer } \\
\text { bis zum } \\
\text { Erwachen }\end{array}$} & \multirow{2}{*}{ Operation } & \multirow{2}{*}{$\begin{array}{l}\text { Vor d. } \\
\text { Nar- } \\
\text { kose } \\
\text { (Vol. } \\
\% \text { ) }\end{array}$} & \multicolumn{7}{|c|}{ Nach d. Narkose } \\
\hline & & & & & $\begin{array}{c}30 \\
\text { Min. }\end{array}$ & $\left|\begin{array}{c}3 \\
\text { Std. }\end{array}\right|$ & $\begin{array}{c}6 \\
\text { Std }\end{array}$ & $\left|\begin{array}{c}24 \\
\text { Stdd. }\end{array}\right|$ & $\left|\begin{array}{c}48 \\
\text { Std. }\end{array}\right|$ & $\mid \begin{array}{c}4 \\
\operatorname{Tag}\end{array}$ & $\begin{array}{c}7 \\
\text { Tag. }\end{array}$ \\
\hline - & - & $6^{\mathrm{h}}$ & Exstirpation & 46,7 & 46,8 & & 40,8 & 46,3 & 45,3 & 46,2 & 48,7 \\
\hline $8^{\prime}$ & - & $7 \mathrm{~h} 30^{\prime}$ & $"$ & 48,5 & 49,0 & & 38,4 & 44,9 & 41,9 & 49,2 & 48,6 \\
\hline $5^{\prime}$ & - & $8^{h}$ & Plastische & 49,1 & 46,8 & & 35,4 & 36,8 & 44,3 & 44,0 & 44,7 \\
\hline $15^{\prime}$ & - & $12^{\mathrm{h}}$ & " & 45,0 & 45,4 & & 40,6 & 48,9 & 48,0 & 44,7 & 45,2 \\
\hline $2^{\prime}$ & + & $7^{\mathrm{h}} 30^{\prime}$ & Darmana- & 47,8 & 47,2 & & 40,5 & 40,1 & 42,5 & 43,6 & 42,1 \\
\hline $14^{\prime}$ & - & $12 \mathrm{~h}$ & Rippenresektion & 50,3 & 46,2 & & 45,2 & 51,1 & & 47,8 & 51,4 \\
\hline $7^{\prime}$ & - & $4^{\mathrm{h}} 30^{\prime}$ & Kastration & 51,7 & 51,2 & & 41,3 & 47,3 & & 53,7 & 51,7 \\
\hline $15^{\prime}$ & - & $9^{\mathbf{h}}$ & Appendektomie & 48,6 & 48,6 & & 46,0 & 45,5 & & 48,4 & 49,8 \\
\hline $7 \prime$ & - & $13^{x} \times 30^{\prime}$ & Inzision & 46,5 & 37,9 & & 41,7 & 35,6 & & 48,9 & 46,2 \\
\hline $25^{\prime}$ & - & $8^{h}$ & $\begin{array}{l}\text { Plastische } \\
\text { Operation }\end{array}$ & 50,8 & 52,3 & & 37,8 & 46,2 & & 53,0 & 50,4 \\
\hline $12^{\prime}$ & - & $2^{\text {lh }}$ & Darmana- & 46,3 & 45,3 & & 38,7 & 45,1 & 39,9 & 46,8 & 47,3 \\
\hline $20^{\prime}$ & - & $13^{h}$ & Trepanation & 49,7 & 50,2 & & 31,9 & 49,1 & 40,3 & 36,9 & 40,8 \\
\hline $10^{\prime}$ & - & $6^{\mathrm{h}}$ & Exstirpation & 50,2 & 49,1 & & 43,2 & 45,1 & 48,3 & 351,6 & 50,7 \\
\hline $15^{\prime}$ & + & $6^{h}$ & , & 49,6 & 50,4 & & 44,8 & 52,7 & 52,5 & $5 \tilde{5} \tilde{0}, 4$ & 49,3 \\
\hline $15^{\prime}$ & - & $11^{\mathrm{h}}$ & $\begin{array}{c}\text { Whitehead'sche } \\
\text { Operation }\end{array}$ & 42,7 & 43,5 & & 31,0 & 41,4 & 47,0 & 42,5 & 41,7 \\
\hline - & - & $5^{\mathrm{b}} 30^{\prime}$ & Exstirpation & 43,7 & 40,6 & 43,3 & $38, \bar{a}$ & 46,9 & 49,0 & 46,6 & 44,0 \\
\hline - & - & $6^{h}$ & Herniotomie & 48,9 & 46,5 & & 41,8 & 50,6 & 49,5 & 50,9 & 49,6 \\
\hline - & - & $6^{\mathrm{h}} 30^{\prime}$ & Trepanation & 42,7 & 44,0 & 44,0 & 38,3 & 39,1 & 43,4 & 42,3 & 42,8 \\
\hline- & + & $7^{\mathrm{h}} 30^{\prime}$ & Rippenresektion & 48,7 & 46,9 & & 40,3 & 43,2 & 50,9 & 99,5 & 50,7 \\
\hline - & - & $8^{h}$ & Exstirpation & 51,7 & 48,0 & & 44,3 & 51,9 & 47,8 & 851,4 & 49,7 \\
\hline - & - & $6^{\mathrm{h}}$ & $"$, & 46,8 & 45,4 & & 37,0 & 49,7 & 51,6 & 54,4 & 52,5 \\
\hline - & - & $9^{h}$ & Inzision & 48,0 & 46,2 & & 38,3 & 37,6 & 45,1 & 43,9 & 43,4 \\
\hline - & - & $13^{\mathrm{h}} 30^{\prime}$ & Exstirpation & 47,3 & 45,5 & & 38,7 & 43,9 & $\pm 0,2$ & 44,2 & 47,1 \\
\hline- & - & $8^{\text {h }} 30^{\prime}$ & , & 47,6 & 44,7 & & 42,0 & 50,8 & 48,7 & 48,7 & 45,0 \\
\hline 一 & 一 & $9^{h}$ & Inzision & 50,3 & 49,4 & & 43,7 & 50,8 & 50,0 & 53,5 & 53,0 \\
\hline - & - & $6^{h}$ & Laparotomie & 46,4 & 44,2 & & 39,7 & 43,0 & 48,2 & 52,6 & $\tilde{5} 2,0$ \\
\hline 一 & - & $8^{\mathrm{h}} 30^{\prime}$ & Kastration & 49,6 & 47,4 & & 43,5 & 49,7 & 52,2 & 51,8 & 52,9 \\
\hline - & - & $4^{h}$ & Rippenresektion & 46,3 & 44,2 & & 42,3 & 49,6 & 48,9 & 48,2 & 49,2 \\
\hline
\end{tabular}

IIb...... Die Gruppe, die am Anfang der Operation zwar ruhig war, am Ende aber unruhig wurde.

III......Die Gruppe, die die Inhalationsnarkose nötig war. 
Tabelle 2. $\mathrm{CO}_{2}$-Gehalt im Blut.

\begin{tabular}{|c|c|c|c|c|c|c|c|c|c|c|c|}
\hline \multirow[b]{2}{*}{$\dot{\mathrm{z}}$} & Name & \multirow[b]{2}{*}{ Diagnose } & \multirow{2}{*}{ 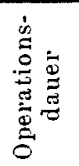 } & \multirow[b]{2}{*}{ Operation } & \multirow{2}{*}{ 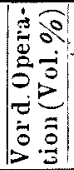 } & \multicolumn{6}{|c|}{ Nach d. Operation } \\
\hline & 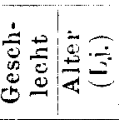 & & & & & 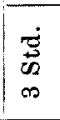 & $\begin{array}{l}\tilde{D} \\
\stackrel{D}{0} \\
0 \\
0\end{array}$ & $\begin{array}{l}\text { Dं } \\
\text { Dू } \\
\text { N }\end{array}$ & $\begin{array}{l}\text { D } \\
0 \\
\infty \\
\infty \\
\forall\end{array}$ & 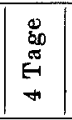 & $\underset{⿱ 乛 龰}{\stackrel{D}{8}}$ \\
\hline 1 & क'T.M. 47 & $\begin{array}{l}\text { Morbus base- } \\
\text { dowi }\end{array}$ & $50^{\prime}$ & Strumektomie & 47,5 & & 36,3 & 46,2 & 47,0 & 48,0 & 44,7 \\
\hline 2 & K.D.36 & $\begin{array}{c}\text { Intervall-A ppen- } \\
\text { djcitis }\end{array}$ & $45^{\prime}$ & $\begin{array}{l}\text { Appendek- } \\
\text { tomie }\end{array}$ & 45,3 & & 43,3 & 41,9 & 42,6 & 41,6 & 46,7 \\
\hline 3 & Q O.T. 20 & Schiefhals & $40^{\prime}$ & $\begin{array}{l}\text { Plastische } \\
\text { Operation }\end{array}$ & 46,1 & & 44,2 & 43,9 & 47,3 & 46,1 & 46,6 \\
\hline 4 & 6 S.K. 26 & $\begin{array}{l}\text { Perityphlitischer } \\
\text { Abszess }\end{array}$ & $1^{1 / 4} 40^{\prime}$ & $\begin{array}{l}\text { Appendek- } \\
\text { tomie }\end{array}$ & 51,1 & & 45,9 & 46,2 & 46,0 & 53,1 & 53,4 \\
\hline 5 & 우 J.T. 26 & $\begin{array}{l}\text { Lymphadenitis } \\
\text { colli tbc. }\end{array}$ & $3^{\ln } 20^{\prime}$ & Exstirpation & 51,1 & & 41,6 & 47,1 & 52,6 & 52,6 & 50,6 \\
\hline 6 & ㅇ.T.T. 20 & $\begin{array}{l}\text { Lymphadenitis } \\
\text { axillaris tbe. }\end{array}$ & $1^{\mathrm{h}} 20^{\circ}$ & ", & 45,4 & & 40,1 & 45,8 & 45,9 & 41,2 & 46,4 \\
\hline 7 & ९.T. 22 & $\begin{array}{l}\text { Lymphadenitis } \\
\text { colli tbe. }\end{array}$ & $1^{\mathrm{h}} 50^{\prime}$ & ", & 50,0 & 41,4 & 40,5 & 46,0 & 46,0 & 50,5 & 50,9 \\
\hline 8 & o S.B. 32 & $\begin{array}{c}\text { Tumor an d. } 1 . \\
\text { Brust }\end{array}$ & $2^{\prime} 20^{\prime}$ & " & 49,1 & 45,1 & 43,2 & 46,1 & 48,6 & 51,8 & 51,1 \\
\hline 9 & q E.D. 24 & $\begin{array}{l}\text { Aktiomykose } \\
\text { d. Gesichtes }\end{array}$ & $45^{\prime}$ & Inzision & 44,5 & & 38,8 & 44,1 & 45,1 & 45,7 & 44,7 \\
\hline
\end{tabular}

(2) Einfluss der Operation mit lokaler Anästhesie auf die Alkalireserve.

Wie aus Tabelle 2 zu ersehen, zeigte die Alkalireserve in allen Fällen 6 Stunden nach dem Operationsanfang ihr Minimum, d.h. sie nahm um 2-10 Vol.\% ab. Nach 24 Stunden war sie zwar bei manchen Fällen schon fast wiederhergestellt, blieb aber im allgemeinen noch tief. Dann aber neigte sie nach 48 Stunden -4 Tagen zur Alkalosis und kehrte gewöhnlich nach 4-7 Tagen wieder zum ursprünglichen Wert zurück. Diese Veränderungen hangen natürlich vom allgemeinen Zustand des Kranken, von der Art und Weise der Operation und dessen Dauer und Verlauf ab. Bei menem Versuch waren sie von dem Allgemeinzustand deutlich beeinflusst, wie z. B. 2. und 4. Fall nach Laparotomie und beim 6., dessen Fieber nach 4 Tagen sehr hoch stieg, die Alkalireserve stark abnahm. Bei den Fällen mit Laparotomie wurde sie meistens ganz langsam wieder normal.

(3) Einfluss der Avertinnarkose auf die

Alkalireserve des Bluts.

(i) Befund nach 30 Minuten. Bei fast $2 / 3$ der 66 Fällen die auf Tabelle 1, wiedergegeben sind, d. h. bei 47, sank die Alkalireserve, und 
żwar nur stets um weniger als 3 Vol.\%, mit Ausnahme von 6 Fällen. Sie blieb aber bei 2 Fällen unverändert und stieg bei 17 nur um 1-2 Vol.\%. Was die Avertindosis betrifft, so wich die Alkalireserve im allgemeinen dadurch nicht besonders stark voneinander ab, wie in Tabelle 1 wiedergegeben, aber sie sank um 5 Vol.\% beim 8 . und 11. Falle, denen ich eine verhältnismässig grosse Dosis Avertin gegeben hatte, und bei denen wegen Atemstörung Zyanose oder Asphyxie eintrat. Aber beim 37. und 38., die an Wirbelkaries mit Senkungsabszess litten, sank die Alkalireserve auch um 5 Vol.\%, obgleich ich ihnen nur $0,08 \mathrm{~g}$ pro kg zugeführt hatte. Weiter sank sie beim 47. mit Bronchialfistel bei einer Gabe von $0,12 \mathrm{~g}$ pro $\mathrm{kg}$ um $9,4 \mathrm{Vol} \%$. Das alles beruhte m. E. auf der Störung der Atemtätigkeit. Kurz, 30 Minuten nach der Narkose waren die Schwankungen der Alkalireserve ohne Rücksicht auf das Mengenverhältnis des Avertins und den Al]gemeinzustand gar nicht besonders gross, während sie bei den Fällen mit Atemstörung verhältnismässig sehr stark beeinflusst wurde.

(ii) Befund nach 3 Stunden. Hier sank die Alkalireserve im. allgemeinen um 2,9-5,8 Vol.\%, durchschnittlich um 3,5 Vol.\% tiefer als vor der Narkose, beim 17. Falle noch tiefer, d.h. um 9,4 Vol. \%. Nur beim 56. Falle stieg sie aber höher als nach 30 Minuten und besonders bei diesem sogar höher als vor der Narkose, was als grosse Ausnahme zu bezeichnen ist. So sank die Alkalireserve 3 Stunden nach der. Narkose im allgemeinen tiefer als nach 30 Minuten.

(iii) Befund nach 6 Stunden. In dieser Zeit sank die Alkalireserve bei 66 Fällen ohne Ausnahme tiefer als vor derNarkose und zeigte den tiefsten Wert im ganzen Verlaufe der Narkose, nur einige Fälle ausgenommen; sie sank nämlich bei 16 Fällen um weniger als 5 Vol. \%, bei 36 Fällen um 5-10 Vol.\% und bei 14 Fällen um 10-15 Vol. \% tiefer, also war die Zahl der Fälle mit einer Senkung von 5-10 Vol. \% am grössten und machte die Mehrheit aller aus. Deshalb also scheint es in der Regel der Azidosis zu neigen. Kurz, 6 Stunden nach der Narkose nahm die Alkalireserve im ganzen Verlaufe der Narkose am stärksten ab, was hauptsächlich auf der Verminderung des Widerstands der. Kranken, namentlich derTuberkulösen berulıte und auf dem Grad der Operation, aber kaum auf der benutzten Avertinmenge, und kaum auf dem Narkosenzustand während der Operation. Auch in den Fällen, bei denen Inhalationsnarkose als Hilfe benutzt wurden, zeigte die Alkalireserve nicht nennenswerte Verminderung, wahrscheinlich, wegen der momentanen Applikation derselben. Es ist bemerkenswert, dass die Trepanation in dieser Richtung deutliche Beeinflussung 
zeigt. Weiter nahm sie bei den Fällen, deren Alkalireserve vor der Narkose vom Anfang an verhältnismässig niedrig gewesen war, nach der Narkose nur ein wenig ab.

(iv) Befund nach 24 Stunden. Nach 24 Stunden stellte sich die Alkalireserve zum grossen Teile wieder her. Von 66 Fällen zeigten 22 schon Alkalosis, aber nur unter 3 vol. \%. Bei den übrigen aber stand sie immer noch niedrig, d.h. bei 28 Fällen blieb die Abnahmeziffer unter $5 \mathrm{Vol} . \%$, bei 10 unter $5-10 \mathrm{Vol} . \%$, und nur bei 6 betrug sie 10-15Vol.\%, also stieg die Kurve der Alkalireserve dabei viel höher als nach 6 Stunden. Bezüglich der Avertinmenge war die Abnahmeziffer in der Ia-Gruppe, besonders bei den Fällen mit $0,13 \mathrm{~g}$ oder noch mehr pro $\mathrm{kg}$, am kleinsten. Alkalosis trat bei vielen Fällen auf, bei denen es sich um verhältnismässig einfache Exstirpation oder um Inzision handelte und eine ziemlich kleine Avertindose benutzt wurde. In manchen Fällen, die verhältnismässig früh erwachten, sah ich besonders Alkalosis eintreten. Im allgemeinen nahm ich wahr, Wiederherstellung der Alkalireserve hauptsächlich vom Mengenverhältnis des Avertins und auch den Operationseingriffen abhängig zu sein scheint.

(v) Befund nach 48 Stunden. Nun stellte sich die Alkalireserve immer mehr wieder her; bei 36 , also mehr als der Hälfte von 59 Fällen, trat nämlich Alkalosis auf und die Alkalireserve nahm, nur 2 Fälle ausgenommen, immer um weniger als 5 Vol.\% zu. Ihre Abnahmeziffer war bei 18 Fällen geringer als 5 Vol.\%, und betrug bei 5 5-10 Vol.\%, also niemals mehr als $10 \mathrm{Vol} \%$, und auch bei den Fällen mit einer Abnahme unter 5 Vol.\% blieben die Schwankungen bei etwa 23 Vol.\%. Daraus ist zu ersehen, dass sich die Alkalireserve hierbei viel deutlicher besserte als nach 24 Stunden. Alkalosis trat zwar bei vielen Fällen auf, besonders bei IIa-Gruppe am häufigsten, ganz wie das auch nach 24 Stunden der Fall war. In der Ia-Gruppe, bei der ich eine verhältnismässig grosse Dosis benutzte, trat sie hingegen am seltensten auf, also stellte sich die Alkalireserve im Gegenteil bei vielen Fällen nicht wiederher. Im allgemeinen blieb die Alkalireserve bei den Fällen mit Laparotomie und Trepanation unter ihrem Anfangswert, besonders deutlich bei lang dauernden Laparotomien.

(vi) Befund nach 4-7 Tagen. Nach 4 Tagen dauerte Alkalosis bei 33 Fällen noch fort, und die Höhe der gestiegenen Alkalireserve blieb unter 5 Vol.\% (3 Fälle ausgenommen). Unter 30 Fällen aber sank die Alkalireserve bei 27 Fällen um weniger als 3 Vol. \%, als ihr Anfangswert, und bei 3 um 5-10 Vol. \%. Nach 7 Tagen stieg sie bei den anderen 31 Fällen wieder auf, aber bei 28 davon um weniger als 5 Vol.\%. 
Bei den anderen 29 Fällen sank sie um weniger als 5 Vol.\%, bei 2 um 5-10 Vol.\% tiefer, als ihr Anfangswert war, aber niemals um mehr als $10 \mathrm{Vol} . \%$. Also blieben sich die Schwankungen der Alkalireserve nach 4 und 7 Tagen ziffermässig fast gleich. Die alles ist so aufzufassen, dass die Alkalireserve hier fast ihren ursprünglichen Wert von vor der Narkose wieder erreichte.

\section{Epikrise.}

Durch Avertinnarkose sank die Alkalireserve des Bluts im allgemeinen, zuweilen neigte nur zur leichten Azidosis, ohne die Organismen dadurch keine nennenswerte Störung zu erleiden. 30 Minuten nach derNarkose, da schon fast $90 \%$ des benutzten Avertins resorbiert und die Operation noch nicht ausgeführt worden war, zeigte die Alkalireserve bei 47 unter sämtlichen 66 Fällen nur geringe Abnabme, d.h. um weniger als $3 \mathrm{Vol} \%$; bei 17 Fällen stieg sie hingegen um weniger als 3 Vol. $\%$ und bei den anderen 2 Fällen blieb sie unverändert. Also nahm die Alkalireserve 30 Minuten nach der Narkose im allgemeinen nur wenig ab, wobei das erste Anzeichen des Übergangs in Azidosis beobachtet wurde. Aber nach 3 Stunden, besonders nach 6 Stunden, wenn sich die Fälle entweder im Nachschlaf oder kurz vor dem Erwachen befanden, nahm die Alkalireserve olnne Ausnahme am stärksten ab. Nach 24 Stunden kehrte sie wieder ungefähr zu ihrem Wert vor der Narkose zurück und zeigte bei einem Teil der Fälle schon Alkalosis, die dann aber nach 48 Stunden, wenn auch nur vorübergehend, bei mehr als der Hälfte aller Fälle auftrat. Erst nach 4-7 Tagen pflegte die Alkalireserve nach verschiedenen Veränderungen wieder zu ihrem ursprünglichen Wert vor derNarkose zurückzukehren. Aber in meinem Versuch beim Menschen verlief es mit der Alkalireserve nicht immer wie beim Tierversuch, weil sie auch von verschiedenen Bedingungen, wie z. B. vom Heilungsverlauf der Operationswunde, von dem Allgemeinzustand (Ernährung, Temperatur) oder anderen Begleiterscheinungen, beeinflusst wurde ; darin liegt eine Schwjerigkeit für die klinische Untersuchung. Denn die Alkalireserve sank 30 Minuten nach Avertinnarkose infolge der verminderten Erregbarkeit des Atmungszentrums im allgemeinen nur ganz wenig, zuweilen jedoch stieg sie, ein wenig, infolge des sogenannten Puffer-Systems. 6 Stunden nach der Narkose war der Höhepunkt der Avertinentgiftung. erst grade vorbei, und klinisch befanden sich die meisten Fälle noch im Nachschlafstadium, weshalb die Alkalireserve hierbei ihren tiefs- 
ten Wert im ganzen Verlauf erreichte. Mit dem Erwachen aus der Narkose besserte sich dann aber die Erregbarkeit des Atmungszentrums allmählich und dies wirkte auf das Säurebasengleichgewicht des Bluts, vielleicht zusammen mit der regulierenden Tätigkeit der Niere, sodass es nach und nach wieder zur. Norm zurückkehrt. Deshalb kam die Alkalireserve bei den Fällen mit verhältnismässig leichtem Operationseingriff zum Teil schon 24-48 Stunden nach der Narkose wieder zu ihrem Anfangswert zurück; im allgemeinen trat sogar Alkalosis auf. Also darf man daraus schliessen, dass die Alkalireserve höchstwahrscheinlich 4-7 Tage nach der Narkose wieder zu ihrem ursprünglichen Wert zurückkehrt. Aber es gab Fälle, bei denen die Alkalireserve nach einer Operation mit Avertin-Vollnarkose, auch nach 7 Tagen noch nicht wieder ihren Anfangswert erreicht hatte, was vielleicht von verschiedenen Umständen nach der Operation und den Widerstand des Individuums abhängig zu sein schien.

Allerdings lässt sich ersehen, dass die Abnahme der Alkalireserve des Bluts bei Avertinnarkose hauptsächlich auf Art und Weise der Operation und verhältnismässig wenig auf der Narkose beruht.

\section{Zweiter Teil. Blutzucker.}

I. Methodik.

Zur Bestimmung des Blutzuckers benutzte ich die Mikromethode ron Haged or n und J en s e nu bestimmte immer den mittleren Wert durch Doppelbestimmung. Blut entnahm ich aus der kubitalen Vene. Das übrige war alles so wie beim Versuch der Alkalireserve. Zum Narkoseversuch benutzte ich 50 Fälle und zur Kontrolle 9 Fälle.

\section{Ergebnisse.}

(1) Blutzucker bei chirurgischen Kranken.

Im allgemeinen wird die physiologische Grenze des Blutzuckers beim Menschen in der Hungerzeit als $50-110 \mathrm{mg} / \mathrm{dl}$ angesehen ( $\mathrm{Sa} \mathrm{k} \mathrm{a-}$ guchi und Watanabe); also nennt man die Blutzuckermenge von mehr als $120 \mathrm{mg} / \mathrm{dl}$. „Hyperglykämie“. Obgleich alle meine $59 \mathrm{Ver}-$ suchsfälle (Tabelle 3 und 4 ), verschiedene $K$ rankheiten zeigten, betrug. der Maximalwert des Blutzuckers beim 6. Fall der Tabelle 1 nur 110 $\mathrm{mg} / \mathrm{dl}$ und der Minimalwert beim 6. Fall der Tabelle $267 \mathrm{mg} / \mathrm{dl}$, bei den anderen blieb der Blutzuckerwert auch immer in den physiologischen Grenzen; der Durchschnittswert aller Fälle betrug $84,5 \mathrm{mg} / \mathrm{dl}$. 
Zwischen der Gruppe mit Intervall-Appendizitis, Hernia inguinalis oder veralteter Knochenfraktur und der von Tuberkulösen zeigte der Blutzuckerwert fast keinen Unterschied, denn er betrug bei beiden Gruppen im Durchschnitt nur etwas mehr als $85 \mathrm{mg} / \mathrm{dl}$; das berubt vielleicht darauf, dass meine Fälle nicht solche Krankheiten enthielten, welche die Regulierung des Blutzuckers zu beeinfliessen pflegen (z. B. Leberkrankheit, maligne Geschwulst, akute Erkrankung u. a.).

\section{(2) Einfluss der Operation mit lokaler Anästhesie} auf den Blutzucker.

Wie in Tabelle 4 wiedergegeben, pflegte der Blutzucker nach der Operation in Lokalnarkose im allgemeinen zu steigen, jedoch mit Ausnahmen. 3-6 oder 24 Stunden nach der Operation, namentlich nach 6 Stunden erreichte der Blutzucker seinen Maximalwert, wobei er aber nur um weniger als $40 \mathrm{mg} / \mathrm{dl}$ stieg. Beim 2. und 8 . Falle erreichte er erst nach 24 Stunden sein Maximum. Jedenfalls wurde er im allgemeinen höher als vor der Operation und kehrte nach 48 Stunden oder 4-7 Tagen zwar wieder beinahe zum Anfangswert zurück, blieb aber noch etwas höher als dieser (ca $10 \mathrm{mg} / \mathrm{dl}$ ). Diese auf der Operation in Lokalnarkose beruhende Veränderung des Blutzckers wurde auch von verschiedenen andern klinischen Bedingungen beeinflusst. Z. B. stieg er besonder's deutlich bei 2 Fällen mit Laparotomie und Rippenkariesoperation. Dies hing vielleicht von Art der Operation und dem Verlauf danach ab. Bei 2 Fällen mit Hernia inguinalis aber stieg der Blutzucker ausnahmsweise nach der Operation ab und sank schon nach 7 Tagen sogar unter den Anfangswert.

(3) Einfluss der Avertinnarkose auf den Blutzucker. (Vgl. Tabelle 3)

(i) Befund nach 30 Minuten. Bei 34, also bei mehr als der Hälfte von 50 Fällen stieg der Blutzucker auf (geringer als $10 \mathrm{mg} / \mathrm{dl}$ ). Er blieb bei 3 Fällen unverändert und sank bei 13 Fällen. Diese Schwankungen der $\mathrm{Zu}$ - oder Abnahme waren aber klinisch nicht besonders bedeutungsvoll, weil sie ca. $10 \mathrm{mg} / \mathrm{dl}$ blieben. Es ist beachtenswert, dass die Abnahme des Blutzuckers bei den durch verlıältnismässig geringe Avertindose nur in seichte Narkose versetzten Füllen und besonder's häufig bei schwächlichen Tuberkulösen erfolgte.

(ii) Befund nach 3 Stunden. Bei 40 von 44 Fällen stieg der Blutzucker auf, d. h. bei $19 \mathrm{um}$ weniger als $10 \mathrm{mg} / \mathrm{dl}$, bei $13 \mathrm{um} 10-20 \mathrm{mg} / \mathrm{dl}$, 
Tabelle

Blutzuc

\begin{tabular}{|c|c|c|c|c|c|c|c|c|c|}
\hline \multirow[b]{2}{*}{$\mathrm{Nr}$. } & \multicolumn{3}{|c|}{ Name } & \multirow[b]{2}{*}{ Diagnose } & \multirow{2}{*}{$\begin{array}{c}\text { Dosis } \\
\text { (pro } \\
\text { kg) }\end{array}$} & \multirow{2}{*}{$\mid \begin{array}{c}\text { Gesamt- } \\
\text { dosis } \\
(2,5 \% \\
\text { Lösg) } \\
\text { cem }\end{array}$} & \multirow{2}{*}{$\begin{array}{c}\text { Zeit- } \\
\text { dauer } \\
\text { biszur } \\
\text { tiefen } \\
\text { Narkose }\end{array}$} & \multirow{2}{*}{$\begin{array}{l}\text { Opera- } \\
\text { tions- } \\
\text { dauer }\end{array}$} & \multirow{2}{*}{$\begin{array}{l}\text { Zustand } \\
\text { während } \\
\text { der } \\
\text { opera- } \\
\text { tion }\end{array}$} \\
\hline & $\begin{array}{l}\text { Ge } \\
\text { lec }\end{array}$ & $\begin{array}{l}\text { seh- } \\
\text { ht }\end{array}$ & $\begin{array}{l}\text { Alter } \\
(L, j .)\end{array}$ & & & & & & \\
\hline 1 & $\delta$ & S.T. & 21 & Intervall-Appendicitis & 0,17 & 374 & $4^{\prime}$ & $45^{\prime}$ & Ia \\
\hline 2 & $\hat{\beta}$ & A.B. & 20 & Lymphadenitis colli tbc. & 0,17 & 334 & $5^{\prime}$ & 211 & $"$ \\
\hline 3 & $\hat{0}$ & O.N. & 20 & Hernia inguin. ext. & 0,17 & 417 & $7^{\prime}$ & $40^{\prime}$ & $"$ \\
\hline 4 & $\delta$ & H.G. & - 24 & $\begin{array}{l}\text { Cystischer Tumor am } \\
\text { Seitenhals }\end{array}$ & 0,16 & 360 & $7^{\prime}$ & $60^{\prime}$ & $"$ \\
\hline 5 & $\hat{\jmath}$ & S.T. & 29 & Osteomyelitis humeri & 0,16 & 290 & $13^{\prime}$ & $55^{\prime}$ & $"$ \\
\hline 6 & s & K.N. & 36 & Epididymitis tbc. & 0,15 & 282 & $5^{\prime}$ & $40^{\prime}$ & 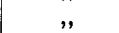 \\
\hline 7 & $\hat{\jmath}$ & U.T. & 26 & Kniegelenkmaus & 0,15 & 362 & $6^{\prime}$ & $15^{\prime}$ & $"$ \\
\hline 8 & 8 & H.N. & 31 & Kotfistel & 0,14 & 332 & $10^{\prime}$ & & $n$ \\
\hline 9 & $\delta$ & K.D. & 27 & KienböckscheKrankheit & 0,14 & 307 & $8^{\prime}$ & $1^{\mathrm{b}} 10^{\prime}$ & $"$ \\
\hline 10 & 8 & S.G. & 23 & Senkungsabszess & 0,13 & 343 & $10^{\prime}$ & $30^{\prime}$ & " \\
\hline 11 & $\delta$ & A. B. & 32 & Oberschenkelgumma & 0,13 & 338 & $10^{\prime}$ & $60^{\prime}$ & , \\
\hline 12 & $\delta$ & S.N. & 35 & Intervall-A ppendicitis & 0,13 & 267 & $8^{\prime}$ & $30^{\prime}$ & , \\
\hline 13 & 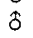 & S.E. & 28 & Bubo inguinalis & 0,12 & 275 & $8^{\prime}$ & $15^{\prime}$ & $"$ \\
\hline 14 & 8 & W.B. & 55 & Hydrocele testis & 0,12 & 269 & $7^{\prime}$ & $20^{\prime}$ & $"$ \\
\hline 15 & $\hat{\delta}$ & T.H. & 62 & Hernia inguin. ext. & 0,11 & 245 & $20^{\prime}$ & $30^{\prime}$ & $"$ \\
\hline 16 & $\uparrow$ & H.Y. & 59 & Hautkrebs am liopf & 0,10 & 162 & $10^{\prime}$ & $2^{\mathrm{h}}$ & , \\
\hline 17 & 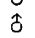 & 0.13 & 20 & Hernia inguin. ext. & 0,16 & 327 & $6^{\prime}$ & $45^{\prime}$ & IIb \\
\hline 18 & $\delta$ & W.M. & .22 & Fractura tibiae & 0,16 & 301 & $27^{\prime}$ & $2^{\mathrm{h}}$ & $\mathrm{Ib}$ \\
\hline 19 & $\delta$ & H.Y. & 31 & Kotfistel & 0,16 & 377 & $9^{\prime}$ & $50^{\prime}$ & " \\
\hline 20 & $\hat{o}$ & W.M. & 35 & Intervall-Appendicitis & 0,15 & 303 & $10^{\prime}$ & $45^{\prime}$ & $"$ \\
\hline 21 & $\delta$ & A.B. & 26 & Intervall-A ppendicitis & 0,15 & 306 & $10^{\prime}$ & $50^{\prime}$ & $"$ \\
\hline 22 & $\hat{\delta}$ & E.D. & 20 & Femursarkom & 0,15 & 348 & $30^{\prime}$ & $60^{\prime}$ & $\mathrm{IIb}$ \\
\hline 23 & 우 & M.K. & 49 & $\begin{array}{l}\text { Lipom in } d . \text { Schulter- } \\
\text { gegend }\end{array}$ & 0,14 & 307 & $7 \prime$ & $15^{\prime}$ & $\mathrm{Ib}$ \\
\hline 24 & $\hat{\delta}$ & A.B. & 19 & Halstumor & 0,14 & 275 & $20^{\prime}$ & $1{ }^{1} 20^{\prime}$ & ", \\
\hline 25 & $\hat{o}$ & N.E. & 22 & Perity phlitischer Abszess & 0,14 & 284 & $4^{\prime}$ & $60^{\prime}$ & $"$ \\
\hline 26 & $\delta$ & S.K. & 26 & Sternumkaris & 0,11 & 222 & seicht & $30^{\prime}$ & , \\
\hline 27 & $\hat{\partial}$ & I.T. & 32 & Lymphadenitis colli tbc. & 0,11 & 282 & seicht & $2^{1} 15^{\prime}$ & $"$ \\
\hline 28 & $\delta$ & O.N. & 15 & Lymphadenitis femoralis & 0,10 & 168 & $8^{\prime}$ & $1^{\text {b }} 20^{\prime}$ & IIb \\
\hline 29 & $\hat{0}$ & O.N. & 55 & Krebs am Gesicht & 0,10 & 260 & $10^{\prime}$ & $40^{\prime}$ & " \\
\hline 30 & $q$ & K.M. & 26 & Rippenkaries & 0,09 & 153 & seicht & $1 \mathrm{l} 30^{\prime}$ & Ib \\
\hline 31 & $\delta$ & O.K. & 17 & Rippenkaries & 0,09 & 182 & seicht & $2^{\mathrm{b}}$ & $"$ \\
\hline 32 & 3 & S.T. & 30 & Fractura humeri & 0,16 & 365 & $10^{\prime}$ & $1^{\mathrm{h}} 50^{\prime}$ & III \\
\hline 33 & $\delta$ & F.N. & 25 & Osteomyelitis tibiae & 0,14 & 380 & $17^{\prime}$ & $50^{\prime}$ & $"$ \\
\hline 34 & $\delta$ & C.B. & 52 & Rippenkaries & 0,12 & 291 & $11^{\prime}$ & $20^{\prime}$ &, \\
\hline 35 & 9 & I.T. & 40 & Sternumkaries & 0,12 & 264 & $8^{\prime}$ & $2 \ln 10^{\prime}$ & , \\
\hline 36 & $\delta$ & M.K. & 20 & Hiiftgelenktuberkulose & 0,11 & 253 & $4^{\prime}$ & $50^{\prime}$ & $"$ \\
\hline 37 & 8 & G.T. & 56 & Coxitis tuberculosa & 0,10 & 174 & seicht & $1^{1} 25^{\prime}$ & $"$ \\
\hline 38 & 官 & A.B. & 25 & KienböckscheKrankheit & 0,10 & 206 & $40^{\prime}$ & $1 \mathrm{~h} 20^{\prime}$ & $"$ \\
\hline 39 & $\delta$ & H.N. & 25 & Lymphadenitis colli tbc. & 0,10 & 238 & $7^{\prime}$ & $2^{\mathrm{h}} 30^{\prime}$ & $"$ \\
\hline 40 & 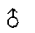 & K.W. & .25 & Intervall-Appendicitis & 0,08 & 153 & $\operatorname{seicht}$ & $1^{\mathrm{h}} 30^{\prime}$ & ", \\
\hline 41 & $\delta$ & M.N. & 36 & Intervall-Appendicitis & 0,15 & 252 & $5^{\prime}$ & $65^{\prime}$ & II a \\
\hline 42 & 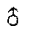 & C.B. & 25 & Rippenkaries & 0,15 & 821 & $8^{\prime}$ & $1 \mathrm{l} 40^{\prime}$ & , \\
\hline 43 & $\hat{o}$ & $0 . Y$ & 28 & $\begin{array}{l}\text { Spontane Gangrän der } \\
\text { beiden Händen }\end{array}$ & 0,13 & 252 & seicht & $50^{\prime}$ & $"$ \\
\hline 44 & $\delta$ & S.D. & 15 & Osteomyelitis tibiae & 0,13 & 255 & $7^{\prime}$ & $50^{\prime}$ & ", \\
\hline 45 & $\hat{\delta}$ & K.B. & 29 & Osteomyelitis humeri & 0,12 & 230 & seicht & $1^{\ln 30^{\prime}}$ & , \\
\hline
\end{tabular}


3.

ker.

\begin{tabular}{|c|c|c|c|c|c|c|c|c|c|c|}
\hline \multirow{2}{*}{$\begin{array}{c}\text { Inhala- } \\
\text { tions- } \\
\text { narkose } \\
\text { (Ather) }\end{array}$} & \multirow{2}{*}{$\begin{array}{c}\text { Zeitdauer } \\
\text { bis zum } \\
\text { Er- } \\
\text { wachen }\end{array}$} & \multirow{2}{*}{ Operation } & \multirow{2}{*}{$\begin{array}{c}\text { Vor d. } \\
\text { Nar- } \\
\text { kose } \\
(\%)\end{array}$} & \multicolumn{7}{|c|}{ Nach d. Narkose } \\
\hline & & & & $\begin{array}{c}30 \\
\text { Min. }\end{array}$ & $\begin{array}{c}3 \\
\text { std. }\end{array}$ & $\begin{array}{c}6 \\
\text { Std. }\end{array}$ & $\begin{array}{c}24 \\
\text { Std. }\end{array}$ & $\begin{array}{c}48 \\
\text { Std. }\end{array}$ & Tag. & $\stackrel{7}{\text { Tag. }}$ \\
\hline- & $15^{\mathrm{h}}$ & Appendektomie & 0,084 & 0,090 & 0,090 & 0,084 & 0,082 & 0,091 & 0,082 & 0,079 \\
\hline - & $12^{11}$ & Exstirpation & 0,090 & 0,095 & & 0,098 & 0,090 & 0,091 & 0,093 & 0,090 \\
\hline- & $14^{\mathrm{h}}$ & Herniotomie & 0,084 & 0,085 & 0,084 & 0,088 & 0,096 & 0,096 & 0,087 & 0,090 \\
\hline - & $12^{\mathrm{h}}$ & Exstiryation & 0,088 & 0,095 & 0,096 & 0,108 & 0,090 & 0,092 & 0,083 & 0,086 \\
\hline- & $13 \mathrm{~h}$ & Aufmeisselung & 0,088 & 0,088 & 0,092 & 0,089 & 0,095 & 0,087 & 0,110 & 0,106 \\
\hline- & $11^{\mathrm{h}}$ & Exstirpation & 0,110 & 0,096 & 0,132 & 0,121 & 0,120 & 0,109 & 0,095 & 0,098 \\
\hline- & $16^{11}$ & & 0,088 & 0,091 & 0,072 & 0,100 & 0,100 & 0,097 & 0,095 & 0,084 \\
\hline - & $8^{h}$ & ohne Operation & 0,088 & 0,089 & 0,092 & 0,099 & 0,090 & 0,088 & 0,087 & 0,097 \\
\hline - & $11^{\mathrm{h}}$ & Exstirpation & 0,082 & 0,089 & 0.092 & 0,096 & 0,100 & 0,092 & 0,086 & $0,08 t$ \\
\hline- & $10^{\mathrm{h}}$ & Inzision & 0,088 & 0,090 & 0,098 & 0,120 & & 0,111 & 0,090 & 0,085 \\
\hline - & $11^{h} 30^{\prime}$ & Exstirpation & 0,076 & 0,078 & & 0,076 & 0,095 . & 0,086 & 0,083 & 0,069 \\
\hline- & $8^{h}$ & Appendektomie & 0,076 & 0,089 & 0,107 & 0,102 & $0,10 \mathrm{c}$ & 0,076 & 0,108 & 0,082 \\
\hline - & $8^{\mathbf{h}}$ & Inzision & 0,081 & 0,083 & 0,084 & 0,088 & 0,102 & 0.081 & 0,090 & 0,079 \\
\hline - & $11^{h}$ & $\begin{array}{c}\text { Winkelma nn'- } \\
\text { sche Operation }\end{array}$ & 0,078 & 0,082 & 0,092 & 0,105 & 0,091 & 0,139 & 0,082 & 0,078 \\
\hline- & $14^{h}$ & Hernioto & 0,085 & 0,085 & 0,095 & 0,106 & 0,111 & 0,116 & 0,101 & 0,081 \\
\hline - & $17^{1}$ & Exs & 0,071 & 0,090 & 0,143 & 0,141 & 0,118 & 0,113 & 0,104 & $0,09 t$ \\
\hline- & $11^{h}$ & Hern & 0,084 & 0,078 & 0,097 & 0,095 & 0,088 & 0,090 & 0,077 & 0,080 \\
\hline 一 & $10^{h}$ & $\begin{array}{r}\text { Plastis } \\
\text { Oper }\end{array}$ & 0,095 & 0,098 & 0,109 & $0,100 \mid$ & 0,107 & 0,102 & 0,112 & 0,096 \\
\hline - & $7^{h}$ & Darmre & 0,088 & 0,088 & 0,107 & 0,096 & 0,077 & 0,072 & 0,085 & 0.093 \\
\hline - & $y^{1}$ & Appendektomie & 0,081 & 0,082 & 0,096 & 0,108 & 0,075 & 0,073 & 0,081 & 0,090 \\
\hline - & $10^{\mathrm{h}}$ & , & 0,087 & 0,090 & 0,088 & 0,088 & 0,090 & 0,094 & 0,101 & 0,088 \\
\hline- & $8^{12}$ & Exstirpation & 0,081 & 0,076 & 0,074 & 0,093 & 0,087 & 0,077 & 0,075 & 0,082 \\
\hline - & $13^{h}$ & $"$ & 0,086 & 0,093 & 0,098 & 0,102 & 0,141 & 0,112 & 0,104 & 0,104 \\
\hline - & $16^{\mathrm{h}}$ & & 0,090 & 0,093 & 0,111 & 0,123 & 0,128 & 0,102 & 0,111 & 0,091 \\
\hline- & $3^{11}$ & Appendektomie & 0,081 & 0,093 & 0,091 & 0,082 & & 0,081 & 0,081 & 0,096 \\
\hline - & $4^{\mathrm{l}_{1}}$ & Inzision & 0,092 & 0,084 & & 0,083 & 0,118 & 0,099 & 0,114 & 0,082 \\
\hline - & $5^{\text {hl }}$ & Exstirpation & 0,087 & 0,091 & 0,096 & 0,105 & 0,096 & 0,092 & 0,091 & 0,089 \\
\hline - & $4^{1 \mathrm{i}}$ & ", & 0,077 & 0,092 & & 0,100 & 0,102 & 0,111 & 0,093 & 0,092 \\
\hline- & $13130^{\prime}$ & & 0,082 & 0,088 & 0,088 & 0,082 & 0,146 & 0,082 & 0,082 & 0,088 \\
\hline- & $5^{l t}$ & Rippenresektion & 0,087 & 0,097 & 0,102 & 0,082 & 0,094 & 0,086 & 0,088 & 0,091 \\
\hline- & $6^{\mathrm{I1}}$ & & 0,092 & 0,088 & 0,108 & 0,115 & 0,120 & 0,101 & $0,123 \mid$ & 0,090 \\
\hline $4^{\prime}$ & $16^{11}$ & $\begin{array}{l}\text { Plastische } \\
\text { Operation }\end{array}$ & 0,083 & 0,095 & 0,103 & 0,110 & 0,096 & 0,115 & 0,101 & 0,108 \\
\hline $17 \prime$ & $10^{\mathrm{h}}$ & A ufmeisselung & 0,083 & 0,070 & 0,087 & 0,092 & 0,083 & 0,076 & 0,099 & 0,087 \\
\hline $6^{\prime}$ & $17130^{\prime}$ & Rippenresektion & 0,091 & 0,095 & 0,099 & 0,096 & 0,113 & 0,101 & 0,088 & 0.102 \\
\hline $7^{\prime}$ & $3^{\text {hl }}$ & & 0,086 & 0.093 & 0,119 & 0,116 & & 0,096 & 0,109 & 0,085 \\
\hline $14^{\prime}$ & $6^{\mathrm{h}}$ & Gelenkresektion & 0,082 & 0,081 & 0,088 & 0,118 & 0,102 & 0,091 & 0,095 & 0,094 \\
\hline $4^{\prime}$ & $8^{3} 30^{\prime}$ & Exartikulation & 0,071 & 0,067 & 0,104 & 0,107 & 0,066 & 0,148 & 0,095 & 0,100 \\
\hline $8^{\prime}$ & $7 \mathrm{lt}$ & Exstirpation & 0,088 & 0,092 & 0,105 & 0,104 & 0,092 & 0,092 & 0,107 & 0,092 \\
\hline $6^{\prime}$ & $6^{11}$ & & 0,086 & 0.097 & & 0,117 & 0,129 & 0,116 & 0,111 & 0,093 \\
\hline $8^{\prime}$ & $5^{\mathrm{lt}}$ & Appendektomie & 0,091 & 0,099 & 0,102 & 0,112 & 0,107 & 0,107 & 0,098 & 0,099 \\
\hline - & $8^{11}$ & Appendektomie & 0,083 & 0,094 & 0,096 & 0,093 & 0,094 & 0,107 & 0,102 & $0,07 \mathrm{~S}$ \\
\hline- & $7^{14}$ & Rippenresektion & 0,084 & 0,070 & & 0,095 & 0,121 & 0,080 & 0,081 & 0,071 \\
\hline 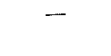 & $3^{h}$ & $\begin{array}{l}\text { Periarterielle } \\
\text { Sympathektomie }\end{array}$ & 0,072 & 0,078 & 0,082 & 0,076 & & 0,079 & 0,070 & 0,068 \\
\hline - & $9^{\prime \prime} 30^{\prime}$ & Inzision & 0,072 & 0,078 & 0,091 & 0,102 & 0,096 & 0,089 & 0,074 & 0,074 \\
\hline 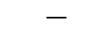 & $9]^{\prime} 30^{\prime}$ & $"$ & 0,096 & 0,103 & 0,091 & 0,098 & 0,144 & $\mid 0,107$ & 0,114 & 0,088 \\
\hline
\end{tabular}




\begin{tabular}{|c|c|c|c|c|c|c|c|c|}
\hline \multirow[b]{2}{*}{$\mathrm{Nr}^{2}$} & \multicolumn{2}{|c|}{ Name } & \multirow[b]{2}{*}{ Diagnose } & \multirow{2}{*}{$\begin{array}{c}\text { Dosis } \\
\text { (pro } \\
\text { kg) }\end{array}$} & \multirow{2}{*}{$\begin{array}{c}\text { Gesamt- } \\
\text { dosis } \\
(2,5 \% \\
\text { Lösg) } \\
\text { cem }\end{array}$} & \multirow{2}{*}{$\begin{array}{c}\text { Zeit- } \\
\text { dauer } \\
\text { bis zum } \\
\text { tiefen } \\
\text { Narkose }\end{array}$} & \multirow{2}{*}{$\begin{array}{l}\text { Opera- } \\
\text { tions- } \\
\text { dauer }\end{array}$} & \multirow{2}{*}{$\begin{array}{l}\text { Zustand } \\
\text { während } \\
\text { der } \\
\text { Opera- } \\
\text { tion }\end{array}$} \\
\hline & $\begin{array}{l}\text { Gesch- } \\
\text { lecht }\end{array}$ & $\begin{array}{c}\text { Alter } \\
(\mathrm{L}, \mathrm{i} .)\end{array}$ & & & & & & \\
\hline 46 & $\delta y . z$ & 46 & Mammakrebs & 0,11 & 216 & $12^{\prime}$ & $1^{l} 20^{\prime}$ & $"$ \\
\hline 47 & S.T & 35 & Empyema thoracis & 0,11 & 254 & $45^{\prime}$ & $\mathrm{l}^{\mathrm{b}} 30^{\prime}$ & , \\
\hline 48 & o K.T & 37 & Rippenkaries & 0,09 & 214 & seicht & $\ln ^{\prime \prime} 20^{\prime}$ & $"$ \\
\hline 49 & $\delta$ T.H & 52 & Helnia inguin. ext. & 0,08 & 163 & $5^{\prime}$ & $1^{1} 20^{\prime}$ & , \\
\hline 50 & I I.T. & 22 & Epididymitis tbe. & 0,08 & 136 & seicht & $1^{11} 40^{\prime}$ & $"$ \\
\hline
\end{tabular}

Anmerkungen.

Ia...... Die Gruppe, die während der Operation ganz ruhig war.

lb...... Die Gruppe, die zwar am Anfang der Operation ganz ruhig war, am Ende aber etwas stöhnte.

IIa..... Die Gruppe, die schon vom Anfang der Operation an unruhig war, aber keine Inhalationsnarkose bedarf.

Tabelle 4.

Blutzucker.

\begin{tabular}{|c|c|c|c|c|c|c|c|c|c|c|c|c|}
\hline \multirow[b]{2}{*}{ 究 } & \multicolumn{2}{|c|}{ Name } & \multirow[b]{2}{*}{ Diagnose } & \multirow{2}{*}{ 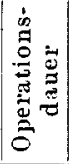 } & \multirow[b]{2}{*}{ Operation } & \multirow{2}{*}{$\left|\begin{array}{rr|} & 0 \\
& 0 \\
0 & 0 \\
0 & 0 \\
0 & 3 \\
0 & 0 \\
0 & 0 \\
0 & 0\end{array}\right|$} & \multicolumn{6}{|c|}{ mach d. Operation } \\
\hline & $\left|\begin{array}{ll}1 & 0 \\
0 & 2 \\
0 & 0 \\
0 & 0 \\
0 & 0 \\
1 & 0\end{array}\right|$ & 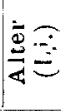 & & & & & $\begin{array}{l}\vec{D} \\
\mathbb{D} \\
\infty\end{array}$ & $\begin{array}{l}\stackrel{d}{J} \\
0,2 \\
0\end{array}$ & 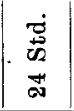 & $\begin{array}{l}\dot{D} \\
i 2 \\
\infty \\
\infty\end{array}$ & 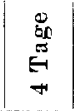 & 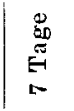 \\
\hline 1 & \multicolumn{2}{|c|}{ \& S.T. 23} & $\begin{array}{l}\text { Hämorrhoidal- } \\
\text { knoten }\end{array}$ & $45^{\prime}$ & Inzision & 0,081 & 0,096 & 0,125 & 0,104 & 0,116 & 0,093 & 0,092 \\
\hline 2 & \multicolumn{2}{|c|}{ \& Z.MI. 24} & $\begin{array}{l}\text { Hernia inguin. } \\
\text { ext. }\end{array}$ & $50^{\prime}$ & Herniotomie & 0,106 & 0,106 & 0,090 & 0,120 & 0,099 & 0,106 & 0,095 \\
\hline$\dot{3}$ & \multicolumn{2}{|c|}{ \& M.K.56 } & Mundlippenkrebs & $35^{\prime}$ & Exstilpation & 0,102 & 0,134 & 0,120 & $0,1 \cap 3$ & 0,104 & 0,104 & 0,108 \\
\hline 4 & \multicolumn{2}{|c|}{ \% М.K. 26} & $\begin{array}{l}\text { Hernia inguin. } \\
\text { ext. }\end{array}$ & $60^{\prime}$ & Herniotomie & 0,075 & 0,066 & 0,086 & 0,070 & 0,068 & 0,076 & 0,067 \\
\hline 5 & \multicolumn{2}{|c|}{ \& M.K.26 } & Rippenkaries & $2 \mathrm{~h}$ & $\begin{array}{l}\text { Rippenresek- } \\
\text { tion }\end{array}$ & 0,082 & 0,107 & 0,115 & 0,100 & 0,096 & 0,093 & 0,090 \\
\hline 6 & \multicolumn{2}{|c|}{ ㅇ K.D. 32} & Analpoly & $15^{\prime}$ & Kauterisation & 0,067 & 0,074 & 0,101 & 0,086 & 0,082 & 0,139 & 0,076 \\
\hline 7 & \multirow{3}{*}{\multicolumn{2}{|c|}{$\left|\begin{array}{l}\delta \text { K.D. } 55 \\
\text { o.T. } 55 \\
\text { s S.K. } 24\end{array}\right|$}} & $\begin{array}{l}\text { Intervall-Appen- } \\
\text { dicitis }\end{array}$ & $50^{\prime}$ & $\begin{array}{c}\text { Appende- } \\
\text { ktomje }\end{array}$ & 0,084 & 0,108 & 0,111 & 0,093 & 0,129 & 0,109 & 0,101 \\
\hline 8 & & & Epididsmitis tbe. & $45^{\prime}$ & Kastration & 0,084 & 0,086 & 0,084 & 0,108 & 0,091 & 0,082 & 0,080 \\
\hline 9 & & & $\begin{array}{l}\text { Lymphadenitis } \\
\text { colli tbc. }\end{array}$ & $1^{h} 50^{\prime}$ & Exstirpation & 0,092 & 0,096 & 0,082 & 0,087 & 0,084 & 0,089 & 0,077 \\
\hline
\end{tabular}

bei 7 um 20-50 mg/dl und nur beim 16 . Falle um $70 \mathrm{mg} / \mathrm{dl}$. Er sank aber bei 4 Fällen stets um weniger als $10 \mathrm{mg} / \mathrm{dl}$. Was die Avertindosis betrifft, so stieg der Blutzucker uiberhaupt bei den Fällen deutlich auf, wo eine kleine Dosis gegeben wurde. Was der Zustand der Kranken während der Narkose betrifft, so stieg der bei der IaGruppe, die bei der Operation ruhig blieb, verhältnismässig wenig, d.h. im allgemeinen nur um weniger als $10 \mathrm{mg} / \mathrm{dl}$, nur den 16 . Falle ausgenommen; also war die Blutzuckerhöhe dabei fast gar nicht verschieden von der nach 30 Minuten. Aber bei der Ma-und III-Gruppe war 


\begin{tabular}{|c|c|c|c|c|c|c|c|c|c|c|}
\hline \multirow{2}{*}{$\begin{array}{l}\text { Inhala- } \\
\text { tions- } \\
\text { narkose } \\
\text { (Äther) }\end{array}$} & \multirow{2}{*}{$\begin{array}{c}\text { Zeitdauer } \\
\text { bis zum } \\
\text { Er- } \\
\text { wachen }\end{array}$} & \multirow[b]{2}{*}{ Operation } & \multirow{2}{*}{$\begin{array}{c}\text { Vor d. } \\
\text { Nar- } \\
\text { kose } \\
(\%)\end{array}$} & \multicolumn{7}{|c|}{ Nach d. Narkose } \\
\hline & & & & $\begin{array}{c}30 \\
\text { Min. }\end{array}$ & $\begin{array}{c}3 \\
\text { Std. }\end{array}$ & $\begin{array}{c}6 \\
\text { Std. }\end{array}$ & $\begin{array}{c}24 \\
\text { Std. }\end{array}$ & $\begin{array}{c}48 \\
\text { Std. }\end{array}$ & $\left|\begin{array}{c}4 \\
\text { Tage }\end{array}\right|$ & $\begin{array}{c}7 \\
\text { Tage }\end{array}$ \\
\hline - & $10^{\mathrm{h}}$ & $\begin{array}{l}\text { Amputatio } \\
\text { mammae }\end{array}$ & 0,093 & 0,076 & 0,097 & 0,100 & 0,093 & 0,088 & 0,100 & 0,129 \\
\hline- & $4^{11}$ & Rippenresektion & 0,078 & 0,073 & 0,110 & 0,081 & 0,096 & 0,090 & 0,083 & 0,060 \\
\hline 一 & $5^{h}$ & , & 0,082 & 0,095 & 0,078 & 0,115 & 0,116 & 0,105 & 0,109 & 0,122 \\
\hline- & $9^{\text {h }}$ & Herniotomie & 0,080 & 0,071 & 0,087 & 0,104 & 0,114 & 0,091 & 0,083 & 0,065 \\
\hline - & $4^{h}$ & Exstirpation & 0,101 & 0,097 & 0,106 & 0,118 & 0,097 & 0,093 & 0,103 & 0,095 \\
\hline
\end{tabular}

IIb......Die Gruppe, die am Anfang der Operation zwar ruhig war, am Ende aber unruhig wurde.

III......Die Gruppe, die die Inhalationsnarkose nötig war.

die Höhe des gestiegenen Blutzuckers im allgemeinen gross und sogar fast doppelt so gross wie nach 30 Minuten. Was die Art der Operation betrifft, so stieg er besonders stark bei dem gestörten Allgemeinzustand, wie beim 47. mit Empyema thoracis, beim 16. mit Kankroid, beim 12. mit Appendizitis und beim 37. mit Koxitis tbc. usw., bei denen die Operation verhältnismässig langdauernd und schwierig war.

(iii) Befund nach 6 Stunden. Bei 48 von 50 Fällen stieg der Blutzucker, d. h. bei $18 \mathrm{um}$ weniger als $10 \mathrm{mg} / \mathrm{dl}$, bei $12 \mathrm{um} 10-20 \mathrm{mg} / \mathrm{dl}$, bei $11 \mathrm{um} 20-30 \mathrm{mg} / \mathrm{dl}$, bei $6 \mathrm{um} 30-40 \mathrm{mg} / \mathrm{dl}$ und nur bei einem um $80 \mathrm{mg} / \mathrm{dl}$; also war die Höhe der Zunahme hierbei im allgemeinen noch höher als nach 3 Stunden, wie auch die Zahl der Fälle, deren Blutzucker um $20-40 \mathrm{mg} / \mathrm{dl}$ stieg, hier noch mehr als doppelt so viel betrug wie nach 3 Stunden. Mit der Avertindosis stand es fast ebenso wie nach 3 Stunden; bei Benutzung einer verhaltnismässig kleinen Menge stieg der Blutzucker sehr stark. Was die Beziehung der Narkosenzustand mit der Blutzucker anbetrifft, so stieg dieser bei der IaGruppe, in welcher die Kranken verhältnismässig ruhig sclliefen, nur ein wenig auf, während bei Älteren und Schwächlichen ziemlich auffallend (6., 14., 15. und 16.). Bei der Ia-, IIa-und IIb-Gruppe zeigte er keine nennenswerte Veränderung, aber bei 4 Fällen der III-Gruppe, die während der Operation unruhig waren, stieg er um $40 \mathrm{mg} / \mathrm{dl}$ oder etwas darüber. Also war die Zunahme des Blutzuckers bei Schwächlichen, die während der Operation unrubig waren und bei denen der Eingriff gross und lang war, besonders auffallend.

Die Zeit des Erwachens hängt dabei scheinbar nicht mit den Schwankungen des Blutzuckers zusammen. Aber bei Tuberkulose, malignem Tumor oder bei Fällen mit Laparotomie stieg der Blutzucker 
ziemlich stark; das stimmt fast genau den Schwankungen der Alkalireserve zu dieser Zeit überein.

(iv) Befund nach 24 Stunden. Unter 46 Fällen stieg der Blutzucker bei 6 ab, aber bei 40 auf, d.h. bei $12 \mathrm{um}$ weniger als $10 \mathrm{mg} / \mathrm{dl}$, bei $11 \mathrm{um} 10-20 \mathrm{mg} / \mathrm{dl}$, bei $8 \mathrm{um} 20-30 \mathrm{mg} / \mathrm{dl}$, bei $7 \mathrm{um} 30-50 \mathrm{mg} / \mathrm{dl}$ und nur bei 2 um $60 \mathrm{mg} / \mathrm{dl}$ oder etwas darüber; also war die Zunahmeziffer dabei fast gleich wie nach 6 Stunden, und nur bei einigen Fällen stieg der Blutzucker besonders stark auf. Die Zunahme des Blutzukkers hing gar nicht sehr vou der Avertindosis und der Haltung während der Operation ab, sondern sie war bedeutend bei den schwächlichen Tuberkulösen und bei Älteren, denen immer eine verhältnismässig kleine Avertindosis gebraucht wurden. Also wurden die Schwankungen des Blutzuckers nach 24 Stunden nicht besonders von denen nach 6 Stunden.

(v) Befund nach 48 Stunden. Unter 50 Fällen sank der Blutzucker bei 10 noch unter den ursprünglichen Wert von vor der Narkose, stieg aber bei 40 , d. h. bei $22 \mathrm{um}$ weniger als $10 \mathrm{mg} / \mathrm{dl}$, bei $7 \mathrm{um}$ $10-20 \mathrm{mg} / \mathrm{dl}$, bei 4 um $20-30 \mathrm{mg} / \mathrm{dl}$, bei 5 um $30-50 \mathrm{mg} / \mathrm{dl}$, bei einem um etwas melır als $70 \mathrm{mg} / \mathrm{dl}$ und endlich bei einem um etwas mehr als $80 \mathrm{mg} / \mathrm{dl}$. Das weist schon auf fortgeschrittene Wiederherstellung hin, weil der Blutzuckerwert bei 32 Fällen, d. h. bei 10 mit gesunkenem Wert und bei $22 \mathrm{mit}$ um weniger als $10 \mathrm{mg} / \mathrm{dl}$ gestiegenen, beinahe wieder zur Stufe vor der Narkose zurückzukehren schien. Bei den Fällen, deren Blutzucker um mehr als $30 \mathrm{mg} / \mathrm{dl}$ stieg (z. B. 14., 16., 37., 39. und 41.), handelte es sich um hohes Alter, ausgenommen solche Fälle, bei denen es zum Teil vom ungewöhnlichen Verlauf nach der Narkose, wie hohem Fieber, oder vom gestörten Heilungsverlauf der Wunde abhing.

(vi) Befund nach 4-7 Tagen. Die Schwankungen des Blutzukkers nach 4 Tagen waren im Vergleich mit denen nach 48 Stunden in den Hauptziigen fast gleich, nur ein wenig verschieden, in Einzelheiten. Denn er sank bei 11 Fällen noch tiefer als vor der Narkose und stieg bei $18 \mathrm{um}$ weniger als $10 \mathrm{mg} / \mathrm{dl}$, bei $11 \mathrm{um} 10-20 \mathrm{mg} / \mathrm{dl}$, bei $6 \mathrm{um}$ $20-30 \mathrm{mg} / \mathrm{dl}$ und bei $4 \mathrm{um} 30-40 \mathrm{mg} / \mathrm{dl}$. Auch in anderer Hinsicht stimmten die Schwankungen dabei fast mit denen nach 42 Stunden iiberein. Aber nach 7 Tagen änderte sich das Bild; bei 20 Fällen sank der Blutzucker sogar noch tiefer als vor der Narkose, und bei mehr als $2 / 3$ von ihnen um weniger als $10 \mathrm{mg} / \mathrm{dl}$; diese Abnahme bedeutet aber gar nichts Ungewöhnliches. Weiter stieg er bej 20 Fällen um weniger als $10 \mathrm{mg} / \mathrm{dl}$ und bei $5 \mathrm{~nm} 10-20 \mathrm{mg} / \mathrm{dl}$; also kann man sagen 
dass der Blutzucker bei allen diesen Fällen, d. h. im ganzen bei 45, schon beinahe wieder den Anfangswert von vor der Narkose, erreichte. Aber bei den anderen 5 Fällen stieg er ganz ungewöhnlich für den ganzen Verlauf, nämlich um $30-50 \mathrm{mg} / \mathrm{dl}$, was natürlich beweist, dass der Blutzucker je nach den verschiedenen klinischen Bedingungen nach der Operation ziemlich heftig beeinflusst ist. Kurz, 4 Tagen nach der Narkose war die Veränderung des Blutzuckers zwar fast ebenso wie nach 48 Stunden, aber nach 7 Tagen stand es anders damit, da der Blutzuckerwert nur ein wenig vom ursprünglichen Wert vor der Narkose abwich.

\section{Epikrise.}

Nach Avertinnarkose stieg der Blutzucker im allgemeinen fast zu allen Perioden, aber gar nicht erheblich. Nach 30 Minuten veränderte er sich nur sehr wenig, d. h. ca. $10 \mathrm{mg} /$ dl, wobei die Zahl der Fälle mit gestiegenem Blutzucker sogar $68 \%$ aller 50 Fälle betrug, während er bei den anderen im Gegenteil sank; diese Abnahme beruhte vielleicht darauf, dass der Blutzucker bei Kranken schon vor der Narkose infolge psychischer Aufregung gestiegen war. Nach 3,6 und 24 Stunden war der Blutzucker aber stark aufgestiegen und zwar nach 3 Stunden blieb die Steigerung mit Ausnahme einiger Fälle, im allgemeinen, doch verhältnismässig klein, ihre Höhe war von der nach 30 Minuten nicht besonders verschieden. Nach 6 Stunden, d.h. gerade im Stadium des Nachschlafs oder direkt vor dem Erwachen, erreichte der Blutzucker den Maximalwert durch den ganzen Verlauf hindurch. Nach 24 Stunden zeigte die Veränderung fast keinen grossen Unterschied von der nach 6 Stunden, und der Blutzucker erreichte auch hier bei vielen FälJen noch den Maximalwert des ganzen Verlaufs; diese Veränderung: rührte vielleicht nicht nur von der Narkose her, sondern hauptsächlich von der Hemmung des Oxydationsprozesses der Organismen nach der Operation; in diesem Punkt war die Veränderung des Blutzukkers von der der Alkalireserve etwas verschieden, welche verhältnismässig rasch in die Norm zurückkehrte. Nach 48 Stunden -4 Tagen war der Blutzuckerwert sclion in bedeutendem Masse wieder hergestellt, blieb aber noch hoch. Dann aber sank er allmählich und kehrte nach verschiedenen Schwankungen erst nach 7 Tagen fast wieder zum Anfangswert von vor der Narkose zurück. Dass der Blutzucker nach einer Operation in Narkose keine ty pische Kurve bildet, ist ohne Zweifel nicht nur auf den Narkoseeinfluss, sondern auch auf verschierene 
mit der Operation verbundene Bedingungen, wie z. B. Blutung, Fieberanstieg, Muskelbewegung, psychische Einflüsse und Ernährung'szustand zurückzuführen.

Auf die Veränderung des Blutzuckers wirkte die Azidosis, d.h. die Störung des Säurebasengleichgewichts im Blut, sehr stark; es gilt allgemein, dass die Steigerung der H-Konzentration des Bluts auf der Vermehrung des Blutzuckers bezogen werden kann, obgleich diese beiden nicht immer miteinander parallel gehen. Man schreibt indessen die Zunahme des Blutzuckers der sekundären Wirkung der Steigerung der H-Konzentration zu, weil diese die Verzuckerung des Leberglykogens fördere. Atkinson und $\mathrm{Ets}^{32}$ und Wymer $\mathrm{r}^{13: 14}$ berichteten, dass der Blutzucker notwendig steige, wenn dabei Alkali infolge der Einwanderung der durch Narkose erzeugten pathologischen Säure ins Blut abnehmen und das H-Ion folglich zunehme. Obwohl ich über die Beziehung der Alkalireserve zum Blutzucker bei ein und demselben Individuum nicht beobachten konnte, so glaube ich doch so viel behaupten zu können, dass die Alkalireserve des Bluts im Stadium des Nachschlafs oder in der Nähe des Erwachens bedeutend abnehme, während sie nach 24 und 48 Stunden allmählich wieder aufsteige, sogar voribergehend Alkalosis auftrete, und nach 4 oder 7 Tagen wieder zum Anfangswert zurückkehre. Auch mit dem Blutzucker verhielt es sich im allgemeinen fast gleich, ausgenommen dass er bei ziemlich vielen Fällen nach 24 oder 48 Stunden noch die Zunahme zeigt, was wahrscheinlich von der Zerstörung der Gewebszellen bei der Narkose, oder von der im Heilungsprozess der Operationswunde gehemmten Oxydationstätigkeit der Organismen herrührt.

Allerdings könnte man glauben, dass die Alkalireserve des Bluts und der Blutzucker in gewissem Zusammenhang stehen, jedoch beide im Verlauf nach der Operation mit Narkose nichtimmer Hand in Hand gehen; es scheint in der Regel zu sein, dass der Blutzucker im allgemeinen für die Wiederherstellung noch längere Zeit als die Alkalireserve erfordere.

\section{Schluss.}

Bei Operation unter Avertinrektalnarkose gehen die Schwankungen der Alkalireserve nicht immer mit der des Blutzuckes's parallel. Der Einfluss der Avertinnarkose auf die Alkalireserve und den Blutzucker ist verhältnismässig klein, sodass dadurch klinisch keine Störung hervorzurufen scheint. Deshalb ist es zweckmässig, die chirur- 
gischen Kranken mehr der Vollnarkose als der Basisnarkose sich zu. bedienen.

Zuın Schluss möchte ich auch hier meinem hochverehrten Lehrer, Herrn. Prof. Dr. Sh. Sekig u chi, meinen herzlichsten Dank für seine stets freundliche Führung und seine wertvollen Ratschläge aussprechen.

\section{Literatur.}

(1) Achelis, Narkose u. Anästhesie, 1928, 1, 541: Zit. n. Pfistel (8).

(2) A m mon u. Schroeder, Dtsch. Ztschr. Chir., 1930, 222, 145.

(3) Atkinson u. Ets, Jour. biol. chem., 1922, 52, 5.

(4) Buger, Bourne n. Dreyer, Am. jour. surg., 1930, 9, 82.

(5) Dewes, Arch. klin. Chir., 1923, 122, 173.

(6) Fuss u. Derra, Dtsch. Ztsch. Chir., 1939, 235, 201, u. 587.

(7) Haged orn u. Jensen, Biochem. Ztschr., 1923, 135, 46.

(8) Pfistel, Arch. klin. Chir., 1931, 165, 402.

(9) Sköld, Arch. klin. Chir., 1928, 151, 600.

(10) Steinmetzer u. S woboda, Biochem. Ztsch., 1928, 198, 259.

(11) Uno, Nippon Gekagakkai Zasshi, 1924, 25, 45.

(12) Widen horn, Dtsch. Ztschr. Chir., 1932, 235, 573.

(13) Wy mer, Dtsch. Ztschr. Chir., 1926, 195, 356.

(14) Wy mer u. Fus s, Dtsch. Ztschr. Chir., 1928, 211, 282. 